\title{
LE SESSE DEL LAGO DI BOLSENA
}

\section{Domenico Di Filippo}

1. - Il lago di Bolsena è certamente il più interessante dei laghi dell'Italia Centrale: notevole per la vastità, profondità e per la superba bellezza del paesaggio, a cui le pittoresche isole, la Martana e la Bisentina, avanzi di antichi crateri, conferiscono particolare attrattiva.

Questo lago, a differenza degli altri, è stato oggetto di particolari attenzioni, per ricerche e esplorazioni da parte di parecchi studiosi. G. De Agostini nel 1896-97 vi ha eseguito circa 3000 scandagli e ha disegnato la carta batimetrica del lago al 50.000 ; E. Perroni ha dedicato a questo lago e al bacino idrografico del fume Marta, una intera monografia della sua carta idrografica dell'Italia e il Moderni si è interessato della geologia della zona. La Società Geografica Italiana poi, nel 1901, affidava ad un gruppo di scienziati il completamento dello studio del lago sotto tutti i punti di vista scientifici e a $\mathrm{L}$. Palazzo, in particolare, l'ottica lacuale, i rilievi morfologici, la ricerca e natura dclle sesse e in genere la fisica limnologica della regione.

Si riportano i dati dei principali elementi morfologici del lago, secondo G. De Agostini :

\begin{tabular}{|c|c|}
\hline altitudine & $\mathrm{m} \quad 305$ \\
\hline lunghezza & 13,0 \\
\hline larghezza massima & 11,5 \\
\hline " media & 8,8 \\
\hline perimetro & 43 \\
\hline profondità massima & m 146 \\
\hline media & » 77,9 \\
\hline inclinazione media & $2^{\circ}, 5$ \\
\hline volume & $\mathrm{m}^{3} 8992.10^{\circ}$ \\
\hline $\begin{array}{l}\text { superficie del lago senza } \\
\text { le isole }\end{array}$ & $\mathrm{km}^{2} 114,26$ \\
\hline Bisentina & $» \quad 0,17$ \\
\hline Martana & 0,10 \\
\hline
\end{tabular}

Le interessanti e chiare registrazioni delle sesse ottenute dal Pa. lazzo e riportate in una sua nota, che si riproducono nella stessa gran- 


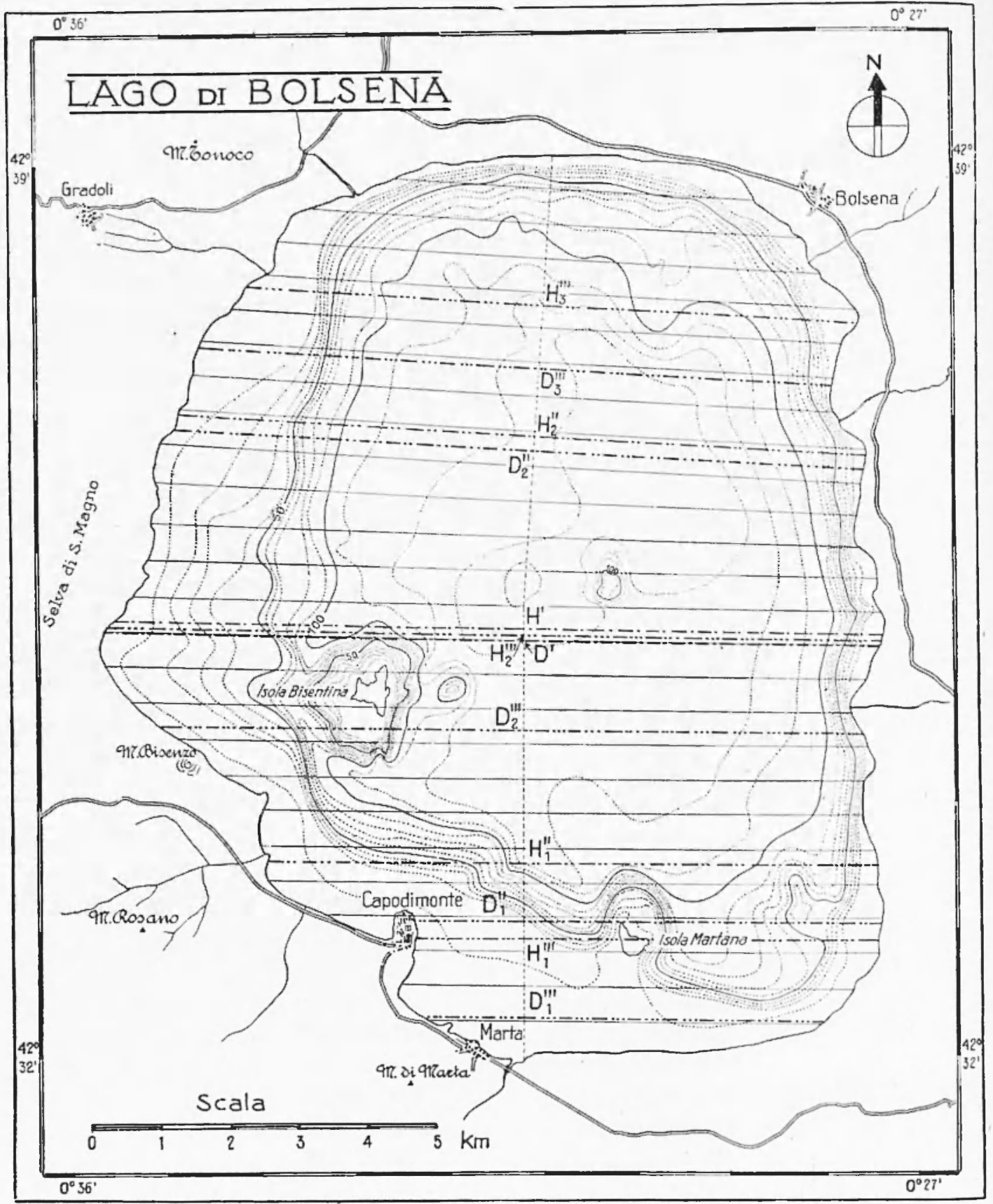

Fig. 1 
dezza in questo lavoro, hanno invogliato al calcolo teorico degli elementi delle prime tre sesse del lago di Bolsena con i metodi di Hidaka e di Defant, onde confrontarli con i dati rilevati dalle osservazioni. Questo lavoro vuole portare un contriluto allo studio sistematico dei laghi italiani, che si sta facendo presso l'I.N.G.

Gli elementi necessari per i calcoli sono stati tratti da una carta batimetrica del lago al 50.000 dell' (Atlante dei laghi italiani ", con le linee di eutuale profondità di 10 in 10 metri.

La linea di valle, con l'origine all'estremità Sud del lago, poco a Est della foce del fiume Marta, segue la linea di massima profondità. Sono state poi tracciate 25 sezioni normali alla linea di valle ed alla distanza di 500 metri l'una dall'altra, data la forma quasi rettangolare del lago.

2. Metodo di Hidaka. - Dalla equazione fondamentale della idrodinamica, il Chrystal ha dedotto una prima teoria per lo studio del moto libero delle sesse, perienendo alla equazione differenziale di una particolare funzione $u$, esprimibile come la somma di semplici fun-

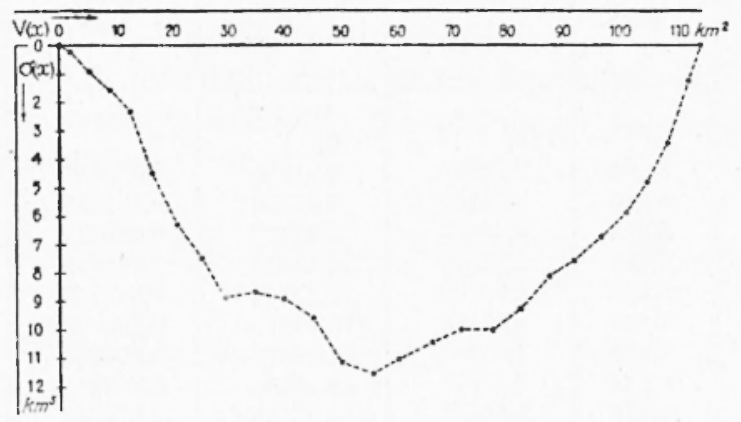

Fig. 2

zioni di $t$. Lo sviluppo di questa teoria è stato esposto dal Caloi nei lavori sulle sesse del Garda.

Se la lunghezza e la forma della sezione trasversale di un lago varia con la profondità, non in maniera brusca, si può procedere al calcolo degli elementi delle sesse conoscendo la funzione $\sigma(x)$, data dal prodotto dell'area $S(x)$ di una sezione trasversale per la lunghezza $b(x)$ di detta sezione alla superficie del lago, e la $V(x)$, area della superficie del lago fra una sezione presa come riferimento e quella corrispondente a $\sigma(x)$.

Si viene così a considerare il lago ridotto, ritenendo retta la linea 
mediana in superficie, uniforme la sua lunghezza e rettangolare una generica sezione trasversale, mentre la sezione longitudinale del lago risulta limitata dalla curva normale di Chrystal, i cui punti hanno per ascissa $V(x)$ e per ordinata la corrispondente $\sigma(x)$.

I dati rilevati dalla prescelta carta batimetrica del lago e le $V(x)$ e $\sigma(x)$ relative ad ogni sezione sono riportate nella tabella $\mathrm{I}$ e la fig. 2 rappresenta il grafico della curva normale.

Alle ipotesi di Chrystal, Hidaka aggiunge la condizione che la funzione $u$ si annulli in corrispondenza del valore 0 e del valore $a$, superficie totale del lago, e ponendo $z=\frac{V}{a}$ perviene alla seguente equazione differenziale

$$
\frac{d^{2} u}{d z^{2}}+\frac{\lambda}{\sigma(z)} u=0
$$

TABella I

\begin{tabular}{|c|c|c|c|c|c|c|}
\hline $\begin{array}{l}\text { N. } \\
\text { Sez. }\end{array}$ & $\underset{\mathrm{km}}{x}$ & $\begin{array}{l}b(x) \\
\mathrm{km}\end{array}$ & $\begin{array}{c}v(x) \\
\mathrm{km}^{2} \\
(\text { parziali) }\end{array}$ & $\begin{array}{c}V(x) \\
\mathrm{km}^{2} \\
\text { (complessive) }\end{array}$ & $\begin{array}{l}S(x) \\
k^{2}\end{array}$ & $\begin{array}{c}\sigma(x) \\
S(x) \cdot b(x) \\
\mathrm{km}^{3}\end{array}$ \\
\hline 0 & 0,0 & 0, & 0 & 0 & & 0 \\
\hline 1 & 0,5 & 5,700 & 2,21950 & 2,21950 & 0,035665 & 0,203290 \\
\hline 2 & 1,0 & 6,540 & 3,25200 & 5,47150 & 0,151750 & 0,992445 \\
\hline 3 & 1,5 & 6,515 & 3,40675 & 8,87825 & 0,240125 & 1,561414 \\
\hline 4 & 2,0 & 7,760 & 3,51000 & 12,38825 & 0,296835 & 2,303440 \\
\hline 5 & 2,5 & 8,850 & 3,98550 & 16,37375 & 0,511500 & 4,526775 \\
\hline 6 & 3,0 & 8,900 & 4,38100 & 20,75475 & 0,708000 & 6,301200 \\
\hline 7 & 3,5 & 8,670 & 4,43900 & 25,19375 & $0,86+250$ & 7,493048 \\
\hline 8 & 4,0 & 9,335 & 4,17075 & 29,36450 & 0,949335 & 8,862042 \\
\hline 9 & 4,5 & 9,950 & 5,00675 & 34,37125 & $0,87+1500$ & 8,701275 \\
\hline 10 & 5,0 & 10,300 & 5,16175 & 39,53300 & 0,864165 & 8.900899 \\
\hline 11 & 5,5 & 10,825 & 5,21200 & 44,74500 & 0,886165 & 9,592736 \\
\hline 12 & 6,0 & 11,210 & 5,45200 & 50,19700 & 1,001500 & 11,226815 \\
\hline 13 & 6,5 & 10,870 & 5,44550 & 55,64250 & 1,061165 & 11,534864 \\
\hline 14 & 7,0 & 10,545 & 5,37600 & 60,01850 & 1,039500 & 10,961528 \\
\hline 15 & 7,5 & 10,275 & 4,98625 & 66,00475 & 1,021000 & 10,490775 \\
\hline 16 & 8,0 & 10,130 & 5,37600 & 71,38075 & 0,996500 & 10,094545 \\
\hline 17 & 8,5 & ] 0,135 & 5,24700 & 76,62775 & 1,019165 & 10,329237 \\
\hline 18 & 9,0 & 9,975 & 5,14375 & 81,77150 & 0,929500 & 9,271762 \\
\hline 19 & 9,5 & 9,360 & 4,85975 & 86,63125 & 0,867835 & 8,122936 \\
\hline 20 & 10,0 & 9,175 & $\begin{array}{l}4,70225 \\
\end{array}$ & 91,33350 & 0,828165 & 7,598414 \\
\hline 21 & 10,5 & 8,850 & 4,44550 & 95,77900 & 0,763165 & 6,754010 \\
\hline 22 & 11,0 & 8,625 & 4,63700 & 100,41600 & 0,690835 & 5,058452 \\
\hline 23 & 11,5 & 7,885 & 3,92300 & 104,33900 & 0,617665 & 4,870289 \\
\hline 24 & 12,0 & 7,225 & $3,7+625$ & 108,08525 & 0,480000 & 3468000 \\
\hline 25 & 12,5 & 5,250 & 3,38950 & 111,47475 & 0,239750 & 1,258687 \\
\hline 26 & 13,0 & 0 & 2,15075 & 113,62550 & & 0 \\
\hline
\end{tabular}


dove

$$
\lambda=\frac{4 \pi^{2} a^{2}}{g T^{2}} .
$$

La [1] rimane soddisfatta solo da particolari valori di $\lambda$. Tenendo conto delle condizioni poste, Hidaka, applicando il calcolo delle variazioni, dà l'integrale

$$
I(u)=\int_{0}^{1}\left\{\left(\frac{d u}{a z}\right)^{-}-\frac{\hat{\lambda}}{\sigma(z)} u^{2}\right\} d z,
$$

in cui $u \dot{e}$ data dall'espressione particolare

$$
u=\sum_{\mathrm{o}}^{\mathrm{m}} A_{\mathrm{i}} z(1-z) z^{1}
$$

Affinché l'integrale [3] risulti minimo è necessario che le derivate parziali di $l(u)$ rispetto alle $A_{\mathrm{i}}$ risultino tutte nulle. Annullando il determinante dei coefficicnte delle $A_{\mathrm{i}}$ nelle relazioni ottenute, si ha l'equazione dei periodi.

Per $m=2$ si perviene alla seguente equazione di $3^{\circ}$ grado in $\lambda$, le cui radici sostituite nella [2] permettono di determinare i periodi delle tre prime sesse:

$$
\begin{aligned}
& \left(I_{0} I_{2} I_{1}-I_{0} I_{3}{ }^{2}-I_{1}{ }^{2} I_{1}+2 I_{1} I_{2} I_{3}-I_{2}{ }^{3}\right) \lambda^{3}-\left[\frac{3}{35}\left(I_{0} I_{2}-I_{1}{ }^{2}\right)+\right. \\
& +\frac{1}{5}\left(-I_{0} I_{3}+I_{1} I_{2}+I_{1} I_{3}\right)+\frac{2}{15} I_{0} I_{4}+\frac{1}{3}\left(-I_{1} I_{4}-I_{2}{ }^{2}+I_{2} I_{3}+\right. \\
& \left.\left.+I_{2} I_{4}-I_{3}{ }^{2}\right)\right) \lambda^{2}+\left(\frac{1}{700} I_{0}-\frac{3}{350} I_{1} \frac{53}{2100} I_{2}-\frac{1}{30} I_{2}+\frac{1}{60} I_{2}\right) \lambda \\
& -\frac{1}{10500}=0
\end{aligned}
$$

con

$$
I_{\mathrm{n}}=\int_{0}^{1} \frac{z^{2}(1-z)^{2} z^{\mathrm{n}}}{\sigma(z)} d z .
$$

Calcolati numericamente gli integrali necessari (tabella II) si sono ottenuti i seguenti risultati

$I_{0}=3,899825, I_{1}=1,840968, I_{2}=1,074459, I_{3}=0,700480, I_{4}=0,489966$. 
Tabella II

\begin{tabular}{|c|c|c|c|c|c|c|}
\hline $\begin{array}{l}\text { N. } \\
\text { Sez. }\end{array}$ & $z=\frac{V}{a}$ & $\begin{array}{c}\boldsymbol{M}= \\
\frac{z^{2}(1-z)^{2}}{\sigma(z)} \Delta z \\
10^{-9}\end{array}$ & $\begin{array}{l}M z \\
10^{-9}\end{array}$ & $\begin{array}{l}M z^{2} \\
10^{-9}\end{array}$ & $\begin{array}{l}M z^{3} \\
10^{-9}\end{array}$ & $\begin{array}{l}M z^{4} \\
10^{-9}\end{array}$ \\
\hline $\begin{array}{r}0 \\
1 \\
2 \\
3 \\
4 \\
5 \\
6 \\
7 \\
8 \\
9 \\
10 \\
11 \\
12 \\
13 \\
14 \\
15 \\
16 \\
17 \\
18 \\
19 \\
20 \\
21 \\
22 \\
23 \\
24 \\
25 \\
26\end{array}$ & $\begin{array}{l}0.01953 \\
0.01815 \\
0.07814 \\
0.10903 \\
0.14410 \\
0.18266 \\
0.22173 \\
0.25843 \\
0.30250 \\
0.34792 \\
0.39379 \\
0.44178 \\
0.48970 \\
0.52821 \\
0.58090 \\
0.62821 \\
0.67 .39 \\
0.71966 \\
0.76243 \\
0.80381 \\
0.84294 \\
0.88375 \\
0.91827 \\
0.95124 \\
0.98107 \\
1.00000\end{array}$ & $\begin{array}{r}35.18667 .1 \\
60.563841 \\
99.474+131 \\
126.554106 \\
117.848737 \\
136.398828 \\
155.271799 \\
152.097 .461 \\
225.47+768 \\
262.646918 \\
272.498120 \\
259.967253 \\
259.427140 \\
218.179 .445 \\
297.6866 .15 \\
255.665511 \\
215.577799 \\
198.735798 \\
172.7 .18201 \\
135.435912 \\
101.548963 \\
72.289610 \\
39.923933 \\
20.456533 \\
8.166171 \\
0 .\end{array}$ & $\begin{array}{r}0.687196 \\
2.916149 \\
7.772932 \\
13.798194 \\
16.982003 \\
24.914610 \\
34.428 .116 \\
39.306547 \\
68.206117 \\
91.380116 \\
107.307035 \\
114.848333 \\
127.041470 \\
115.244565 \\
172.926172 \\
160.611631 \\
145.383512 \\
143.022204 \\
131.708411 \\
108.86+1740 \\
85.599683 \\
63.885943 \\
36.660950 \\
19.459072 \\
8.011585 \\
0 .\end{array}$ & $\begin{array}{r}0.013 .106 \\
0.140387 \\
0.607391 \\
1.504 .175 \\
2.447129 \\
4.550947 \\
7.633783 \\
10.157981 \\
20.632294 \\
31.792884 \\
42.256556 \\
50.737809 \\
62.212185 \\
60.873374 \\
100.452870 \\
100.897883 \\
98.0+55214 \\
102.927456 \\
100.418529 \\
87.506633 \\
72.155413 \\
56.459197 \\
33.661659 \\
18.510258 \\
7.859923 \\
0 .\end{array}$ & $\begin{array}{r}0.000026 \\
0.006760 \\
0.017462 \\
0.164033 \\
0.352631 \\
0.831276 \\
1.692639 \\
2.625127 \\
6.241269 \\
11.061380 \\
16.640209 \\
22.414949 \\
30.465307 \\
32.153925 \\
58.353072 \\
63.385059 \\
66.120712 \\
74.072773 \\
76.562099 \\
70.338706 \\
60.822683 \\
49.895816 \\
30.913246 \\
17.607697 \\
7.711135 \\
0 .\end{array}$ & $\begin{array}{r}0.000001 \\
0.000325 \\
0.003709 \\
0.017884 \\
0.050814 \\
0.151841 \\
0.375309 \\
0.678412 \\
1.787984 \\
3.848475 \\
6.552718 \\
9.902476 \\
14.918861 \\
16.984025 \\
33.897300 \\
39.819128 \\
44.591147 \\
53.307212 \\
58.373241 \\
56.538956 \\
51.269873 \\
44.095427 \\
28.386707 \\
16.749146 \\
7.565163 \\
0 .\end{array}$ \\
\hline & & $I_{0}$ & $\mathrm{I}_{1}$ & $\mathrm{I}_{2}$ & $I_{3}$ & $\mathrm{I}_{4}$ \\
\hline
\end{tabular}

Sostituiti questi valori nella [4], si ha l'equazione

$$
96851,6 \lambda^{3}-79157,8 \lambda^{2}+17255,2 \lambda-952,381=0 \text {, }
$$

che rimane soddisfatta dai valori

$$
\lambda_{1}=0,084683 \quad \lambda_{2}=0,231906 \quad \lambda_{3}=0,500722 .
$$

Dalla [2], tenendo conto che la superficie del lago $a=\mathbf{k m}^{2} 113,6255$, si sono ottenuti i periodi relativi alle sesse uni-bi-trinodali

$$
T_{1}=13^{\mathrm{m}}, 048 \quad, T_{2}=7^{\mathrm{m}}, 885, T_{3}=5^{\mathrm{m}}, 366 .
$$

L'andamento delle ampiezze è dato dalla relazione

$$
\zeta=-\frac{d u}{d z} a
$$


che nel caso considerato, $m=2$, si può scrivere nella forma

$$
\zeta=-A_{0} a\left\{4 \frac{A_{2}}{A_{0}} z^{3}+3\left(\frac{A_{1}}{A_{0}}-\frac{A_{2}}{A_{0}}\right) z^{2}+2\left(1-\frac{A_{1}}{A_{0}}\right) z-1\right\} .
$$

I rapporti delle costanti $A_{\mathrm{i}}$ occorrenti si ottengono limitando le relazioni di condizione ai primi tre termini e risolvendo i sistemi rispetto ai rapporti $\frac{A_{:}}{A_{0}}$ e $\frac{A_{0}}{A_{0}}$, e tenendo conto dei valori degli integrali e delle $\lambda$ relative. Nel caso, si sono ottenute le tre seguenti coppie, rispettivamente per la uni-bi-trinodale

$$
\begin{array}{ll}
\frac{A_{1}}{A_{0}}=-0,5035 & \frac{A_{9}}{A_{0}}=+0,2594 \\
\frac{A_{1}}{A_{0}}=-3,0793 & \frac{-\frac{A_{-}}{A_{0}}}{A_{0}}+1,5443 \\
\frac{A_{1}}{A_{0}}=-6,2222 & \frac{-\frac{-}{-z}}{A_{0}}=+7,0302
\end{array}
$$

\begin{tabular}{|c|c|c|c|}
\hline $\begin{array}{l}\text { N. } \\
\text { Sez. }\end{array}$ & $\begin{array}{c}\text { Uninodale } \\
\eta^{\prime}\end{array}$ & $\underset{\eta "}{\text { Binodale }}$ & $\underset{\eta}{\text { Trinodale }}$ \\
\hline $\begin{array}{r}0 \\
1 \\
2 \\
3 \\
4 \\
5 \\
6 \\
7 \\
8 \\
9 \\
10 \\
11 \\
12 \\
13 \\
14 \\
15 \\
16 \\
17 \\
18 \\
19 \\
20 \\
21 \\
22 \\
23 \\
24 \\
25 \\
26\end{array}$ & $\begin{array}{l}+1, \\
+0,942144 \\
+0,860403 \\
+0,778512 \\
+0,698010 \\
+0,611111 \\
+0,520779 \\
+0,431469 \\
+0,357846 \\
+0,271092 \\
+0,187149 \\
+0.107423 \\
-0,028790 \\
-0,015532 \\
-0.102681 \\
-0,177818 \\
-0,243039 \\
-0,305232 \\
-0,365109 \\
-0,422071 \\
-0,471181 \\
-0,529960 \\
-0,586102 \\
-0,631777 \\
-0,682532 \\
-0,726991 \\
-0,755900\end{array}$ & $\begin{array}{l}+1, \\
+0,815943 \\
+0,638627 \\
+0,4+1235 \\
+0,267357 \\
+0,093889 \\
-0,065098 \\
-0,194100 \\
-0,288667 \\
-0,369704 \\
-0,419660 \\
-0,439029 \\
-0,429754 \\
-0,394372 \\
-0,349775 \\
-0,269566 \\
-0,182694 \\
-0,088243 \\
+0,010057 \\
+0,104997 \\
+0,195980 \\
+0,278829 \\
+0,359.192 \\
+0.421310 \\
+0,473372 \\
+0.513465 \\
+0,535000\end{array}$ & $\begin{array}{l}+1, \\
+0,733028 \\
+0,393524 \\
+0,100660 \\
-0,138682 \\
-0,340018 \\
-0,483288 \\
-0,55+675 \\
-0,562986 \\
-0,509802 \\
-0,397269 \\
-0,240075 \\
-0,046470 \\
+0,158283 \\
+0,318552 \\
+0,512824 \\
+0,6+1219 \\
+0,715448 \\
+0,71+448 \\
+0.634853 \\
+0,172452 \\
+0,230691 \\
-0,123886 \\
-0,513836 \\
-0,970091 \\
-1,458594 \\
-1,808000\end{array}$ \\
\hline
\end{tabular}

Tabella III 
Sostituiti questi valori nella [5], le funzioni che danno l'andamento delle ampiezze sono: per la uninodale

$$
\eta^{\prime}=\frac{\zeta}{A_{0} a}=-\left(1,0376 z^{3}-2,2887 z^{2}+3,0070 z-1\right) ;
$$

successivamente, per la binodale,

$$
\eta^{\prime \prime}=\frac{\zeta}{A_{0} a}=-\left(6,1772 z^{3}-13,8708 z^{2}+8,1586 z-1\right) ;
$$

e infine, per la trinodale,

$$
\eta^{\prime \prime \prime}=\frac{\zeta}{A_{0} a}=-\left(28,1206 z^{2}-39,7570 z^{2}+14,4443 z-1\right) .
$$

Facendo variare $z$, da 0 a 1 , in questa relazione, si ha l'andamento delle ampiezze delle tre sesse riferite al valore per $z=0$. I risultati

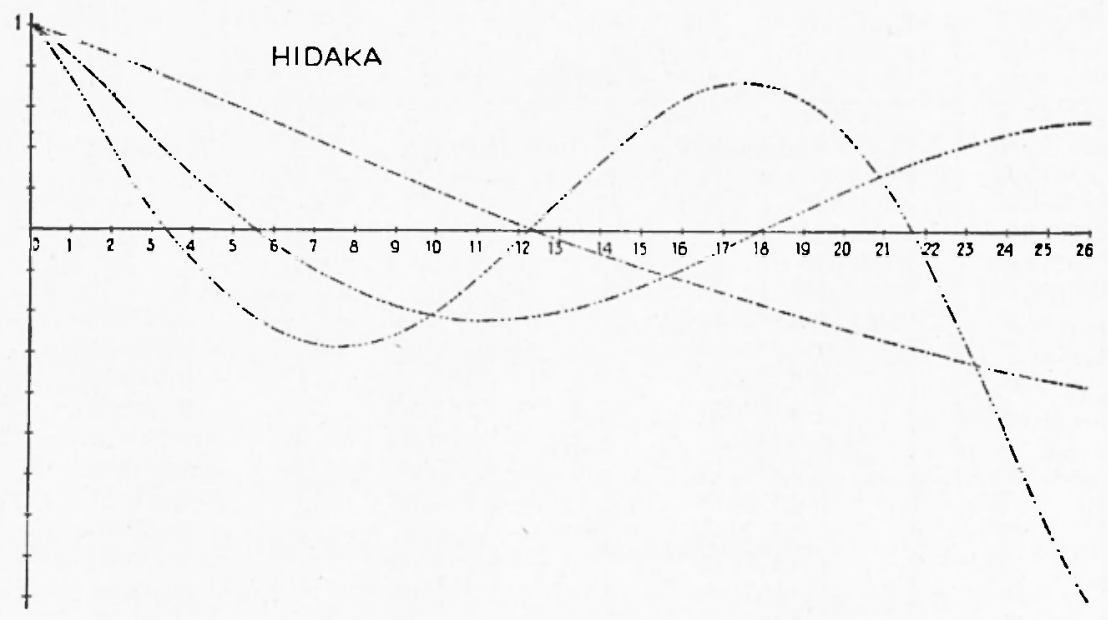

Fig. 3

dei calcoli sono riportati nella tabella III e nella fig. 3 i relativi grafici.

Le posizioni dei nodi, dato che in essi $\frac{d u}{d z}=0$, si hanno in corrispondenza dei valori minori dell'unità che annullano le tre relazioni delle ampiezze. Per la uninodale l'unico valore è

$$
z_{1}^{\prime}=0,460053
$$


che viene a trovarsi distante $\mathrm{km} \mathrm{6,191}$ dall'estremo sud del lago, men. tre per la linodale

$$
z_{1}^{\prime \prime}=0,165911 \quad \text { e } \quad z_{2}^{\prime \prime}=0,715103,
$$

che risultano a $\mathrm{km} 2,783$ e $\mathrm{km} 8,970$ dall'origine. Per la trinodale si hanno $\mathrm{i}$ valori

$$
z^{\prime \prime \prime}{ }_{1}=0,090194, z^{\prime \prime \prime}{ }_{2}=0,452736, z^{\prime \prime \prime}{ }_{3}=0,870870,
$$

distanti da Marta rispettivamente $1,695,6,114$ e $10,842 \mathrm{~km}$.

3. Metodo di Defant. - Dall'equazione di moto e di continuità dell'idrodinamica e facendo riferimento ad una terna di assi cartesiani, con l'asse delle $x$ secondo la linea di valle e la $z$ positiva verso l'alto, il Defant, indicato con $2 \xi$ e $2 \eta$ gli spostamenti orizzontali e verticali degli strati dell'acqua da una sezione Si $x)$ ad una $S(x+d x)$, perviene alle seguenti espressioni

$$
\Delta \eta=\frac{4 \pi^{2}}{g T^{2}} \xi \Delta x \quad \xi=-\frac{1}{S(x)} \int_{0}^{x} \eta b(x) d x,
$$

che permettono di calcolare le ampiezze delle oscillazioni lungo il lago nelle due direzioni. Nel caso dei laghi, si sa che gli spostamenti orizzontali delbbono annullarsi agli estremi. Dai valori riportati nella tabella I delle superfici delle sezioni $S(x)$ trasversali e delle $v(x)$, aree parziali del lago, e partendo dai valori dei periodi delle sesse calcolati col metodo di Hidaka, sono state applicate successivamente le suddette formule alle varie sezioni per il calcolo dei relativi spostamenti. I periodi considerati sono stati successivamente approssimati fino ad ottenere in corrispondenza dell'ultima sezione per una quantità $q$ di acqua spostata un valore più piccolo possibile. I calcoli relativi alle tre prime sesse, con i valori dei periodi più soddisfacenti alle condizioni predette, sono riportati nella tabella IV.

Il metodo permette la contemporanea determinazione dei periodi, delle linee nodali e degli spostamenti verticali per ognni sezione. L'andamento delle ampiezze si rileva dai valori $\eta$, riportati nella tabella IV relativi ad ogni sessa; $\mathbf{i}$ grafici sono rappresentati nella fig. 4 .

I periodi trovati con questo metodo sono, per le tre sesse considerate, rispettivamente

$$
T_{1}=13^{\mathrm{m}}, 15 \quad, \quad T_{2}=8^{\mathrm{m}}, 2 \quad, \quad T_{3}=5^{\mathrm{m}}, 95,
$$

che risultano in ottimo accordo con quelli ottenuti col metodo di 
TABELLA IV

$T_{1}=13^{\mathrm{m}}, 15 \quad \frac{4 \pi^{2} \Delta x}{\mathrm{~g}^{2} T_{1}{ }^{2}}=3,228987.10^{-\mathrm{j}} \quad T_{2}=8^{\mathrm{m}, 2} \quad \frac{4 \pi^{2} \Delta x}{\mathrm{~g}^{2} T_{\mathrm{i}}{ }^{2}}=16.040347 .10^{-3} \quad T_{3}=5^{\mathrm{m}}, 95 \quad \frac{4 \pi^{2} \Delta x}{\mathrm{~g} T_{3}{ }^{2}}=15.77189 \cdot 1 \cdot 10^{-3}$

\begin{tabular}{|c|c|c|c|}
\hline $\begin{array}{c}\text { N. } \\
\text { Sez. }\end{array}$ & $\begin{array}{c}q=\eta v(x) \\
\mathrm{m}^{3}\end{array}$ & $\begin{array}{c}\quad \frac{q}{(x)} \\
\mathbf{c m} \\
\end{array}$ & $\begin{array}{l}\Delta \eta \\
\mathbf{c m}\end{array}$ \\
\hline$u$ & & & \\
\hline 1 & 2219500 & $-62,2319$ & $-20,09$ \\
\hline 2 & 4818173 & $-31,7507$ & $-10,25$ \\
\hline 3 & 7191315 & $-.29,9 \cdot 482$ & $-9,67$ \\
\hline 4 & 9296961 & $-31,3203$ & $-10,11$ \\
\hline 5 & 11284931 & $-22,0624$ & $-7,12$ \\
\hline 6 & 13158247 & $-18,5851$ & $-6,00$ \\
\hline 7 & 14790023 & $-17,1131$ & $-5,53$ \\
\hline 8 & $160925+8$ & $-16,9514$ & $-5,47$ \\
\hline 9 & 17382287 & $-19,8768$ & $-6,42$ \\
\hline 10 & 10380569 & $-21,2697$ & $-6,87$ \\
\hline 11 & 19030505 & $-21,4751$ & $-6,93$ \\
\hline 12 & 19332546 & $-19,3036$ & $-6,23$ \\
\hline 13 & 19294972 & $-18,1828$ & $-5,87$ \\
\hline 14 & 189.12306 & $-18,2225$ & $-5,88$ \\
\hline 15 & 1832 & $-17,9 \cdot 52$ & $-5,79$ \\
\hline 16 & 17341971 & $-.17,4029$ & $--5,62$ \\
\hline 17 & & $-15,7880$ & $-5,10$ \\
\hline 18 & 146014.45 & $-\cdot 15,7089$ & $-5,07$ \\
\hline 19 & 129.48158 & $-14,9201$ & $-4,82$ \\
\hline 20 & 1112180.4 & $-13,4295$ & $-4,3.4$ \\
\hline 21 & 9202237 & $-12,0580$ & -3.89 \\
\hline 22 & 7019601 & $-.10,1610$ & $-3,28$ \\
\hline 23 & $50+14371$ & $-8,1668$ & $-2,64$ \\
\hline 24 & 3059233 & $-6,3734$ & $-2,06$ \\
\hline 25 & 1193313 & $-4,9773$ & $-1,61$ \\
\hline 2 & & & \\
\hline
\end{tabular}

\begin{tabular}{|c|c|c|c|}
\hline $\begin{array}{l}\eta \\
\mathbf{c m}\end{array}$ & $\begin{array}{l}q \\
\mathrm{~m}^{3}\end{array}$ & $\begin{array}{l}\xi \\
\mathrm{cm}\end{array}$ & $\begin{array}{l}\Delta \eta \mid \\
\mathbf{c m}\end{array}$ \\
\hline$+100,00$ & & & \\
\hline $\begin{array}{r}+79,91 \\
\end{array}$ & 2219500 & $-62,2319$ & $-51,68$ \\
\hline$+69,66$ & 3790866 & $-24,9810$ & $-20,7.1$ \\
\hline $\begin{array}{l}+59,99 \\
\end{array}$ & 47304.18 & $-19,6999$ & $-16,36$ \\
\hline$+49,88$ & 512.4270 & $-17,2630$ & $-14,34$ \\
\hline $\begin{array}{r}+42,76 \\
\end{array}$ & 4999922 & $-9,7750$ & $-8,12$ \\
\hline$+36,76$ & 4507.498 & $-6,3665$ & $-5,29$ \\
\hline$+31,23$ & 3773731 & $-4,3665$ & $-3,63$ \\
\hline $\begin{array}{r}+25,76 \\
+\end{array}$ & 2932908 & $-3,1223$ & $-2,59$ \\
\hline$+19,34$ & 1793872 & $-2,0513$ & $-1,70$ \\
\hline$+12,47$ & 531824 & $-0,6154$ & $-0,51$ \\
\hline$+\quad 5,51$ & -769091 & $+0,8679$ & $+0,72$ \\
\hline - 0,69 & -2090656 & $+2,0875$ & $+1,73$ \\
\hline$-6,56$ & -3316438 & $+3,1253$ & $+2,60$ \\
\hline$-12,1.1$ & -1386800 & $-1-4,2201$ & $+3,50$ \\
\hline$-18,23$ & $-52050+4$ & $+5,0980$ & $+4,23$ \\
\hline$-23,85$ & -5859841 & $+5,880.1$ & $+4,88$ \\
\hline$-28,95$ & -6242872 & $+6,1255$ & $+5,09$ \\
\hline-34.02 & -6356549 & $+6,8387$ & $+5,68$ \\
\hline$-38.8 t$ & -6187916 & $+7,1303$ & $+5,92$ \\
\hline$-43,18$ & $--57+6375$ & $+6,9387$ & $+5,76$ \\
\hline$-47,07$ & -5072882 & $+6,6472$ & $+5,52$ \\
\hline$-50,35$ & $--411+414$ & $+5,9557$ & $+4,95$ \\
\hline$-52,99$ & -31093.1 & $+5,0340$ & $+4,18$ \\
\hline$-55,05$ & -1992959 & $+4,1520$ & $+3,45$ \\
\hline$-56,66$ & $\begin{array}{r}865950 \\
-\quad 86303\end{array}$ & $+3,6119$ & $+3,00$ \\
\hline
\end{tabular}

\begin{tabular}{|c|c|c|}
\hline $\begin{array}{l}\eta \\
\mathbf{c m}\end{array}$ & $\begin{array}{l}q \\
\mathrm{~m}^{3}\end{array}$ & $\begin{array}{l}\xi \\
\mathrm{cm}\end{array}$ \\
\hline$+100,00$ & & \\
\hline $\begin{array}{r}+48,32 \\
\end{array}$ & 2219500 & $-62,2319$ \\
\hline $\begin{array}{r}+27,58 \\
\end{array}$ & 2279662 & $-15,0225$ \\
\hline$+11,22$ & 1535628 & $-6,3951$ \\
\hline$-\quad 3,12$ & 414885 & $-1,3977$ \\
\hline$-11,24$ & -945366 & $+1,8182$ \\
\hline$-16,53$ & -2313114 & $+3,2671$ \\
\hline$-20,16$ & -3470361 & $\begin{array}{r}+4,0155 \\
\end{array}$ \\
\hline$-22,75$ & -4293667 & $\begin{array}{r}+4,5228 \\
\end{array}$ \\
\hline$-24,45$ & -4925018 & $\begin{array}{r}+5,6318 \\
\end{array}$ \\
\hline$-24,96$ & -5117551 & $\begin{array}{r}+5,9220 \\
\end{array}$ \\
\hline$-24,24$ & -1825158 & $+5,4450$ \\
\hline$-22,51$ & -4050974 & $\begin{array}{r}+4,0449 \\
\end{array}$ \\
\hline$-19,91$ & -2930290 & $\begin{array}{r}+2,7614 \\
\end{array}$ \\
\hline$-16,41$ & -1589516 & $+1,5291$ \\
\hline$-12,18$ & -225777 & $\begin{array}{r}+0,2211 \\
+\end{array}$ \\
\hline$-7,30$ & +1263375 & $-1,2678$ \\
\hline$-\quad 2,21$ & $\begin{array}{r}+261185 . \\
\end{array}$ & -2.5627 \\
\hline $\begin{array}{l}+3,47\end{array}$ & +3725990 & $-4,0086$ \\
\hline $\begin{array}{r}+\quad 9,39 \\
\end{array}$ & $+4471+76$ & $-5,1524$ \\
\hline$+15,15$ & +4810508 & $-5,8086$ \\
\hline$+20,67$ & +4723821 & $-6,1898$ \\
\hline $\begin{array}{r}25,62 \\
+\end{array}$ & +4180828 & -6.0518 \\
\hline $\begin{array}{r}+29,80 \\
\end{array}$ & +3347191 & $-5,4191$ \\
\hline$+33,25$ & +2230809 & -4.6475 \\
\hline$+36,25$ & $\begin{array}{r}972288 \\
\end{array}$ & $-4,0554$ \\
\hline
\end{tabular}

\begin{tabular}{|c|c|}
\hline$\Delta \eta$ & cm \\
\hline & $+100,00$ \\
\hline -98,15 & $+\quad 1,85$ \\
\hline$-23,69$ & $-21,84$ \\
\hline$-10,09$ & $-31,93$ \\
\hline$-2,20$ & $-34,13$ \\
\hline$+2,91$ & $-31,22$ \\
\hline$+5,15$ & $-26,07$ \\
\hline $\begin{array}{r}6,33 \\
\end{array}$ & $-19,74$ \\
\hline$+7,13$ & $-12,61$ \\
\hline$+8,88$ & $-\quad 3,73$ \\
\hline$+9,34$ & $+\quad 5,61$ \\
\hline$+8,59$ & $+1 \cdot 4,20$ \\
\hline$+6,38$ & $+20,58$ \\
\hline$+4,36$ & $+24,9.4$ \\
\hline 2,41 & $+27,35$ \\
\hline 0,35 & $+27,70$ \\
\hline$-2,00$ & $+25,70$ \\
\hline$-4,01$ & $+21,66$ \\
\hline$-6,32$ & $+15,34$ \\
\hline$-8,13$ & $+7,21$ \\
\hline$-9,16$ & $-\quad 1.95$ \\
\hline$-9,76$ & -11.71 \\
\hline$-9,5.4$ & $-21,25$ \\
\hline$-8,55$ & $-29,80$ \\
\hline 7,33 & $-37,13$ \\
\hline$-6,40$ & $-43,53$ \\
\hline
\end{tabular}


Hidaka; si deve solo notare che le differenze crescono con l'ordine della sessa e sono $0^{\mathrm{m}}, 1 ; 0^{\mathrm{m}}, 3$ e $0^{\mathrm{m}}, 55$.

4. Le osservazioni. - Come si è detto, il Palazzo fu incaricato nel 1901 dalla Società Geografica Italiana, dello studio dei fenomeni di fisica limnologica del lago di Bolsena; e per la ricerca e la natura delle sesse pose un limnografo Sarasin a Bolsena ed un altro a Marta. Il limnografo di Marta, come nota Palazzo, diede risultati, per la

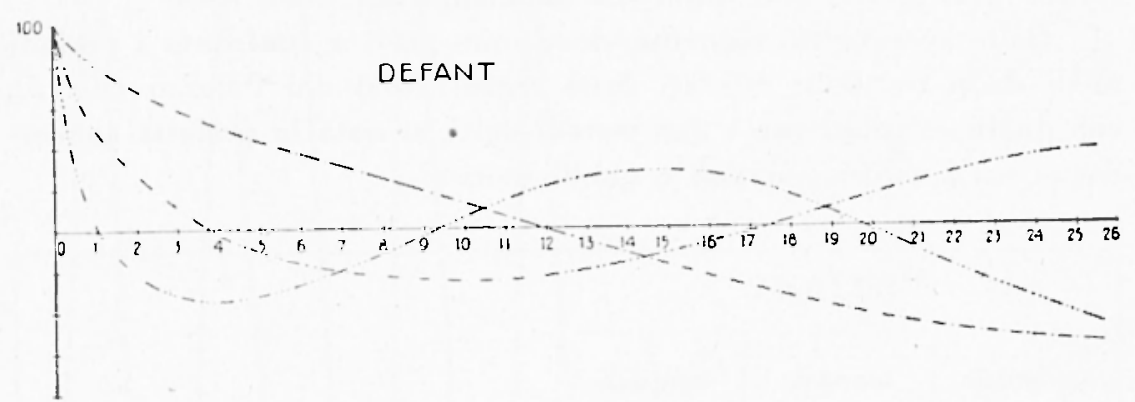

Fig. 4

nitidezza delle sesse registrate, da non potersi immaginare nulla di più tipico, tanto che la sessa uninodale a Marta e possibile osservarla anche ad occhio, riferendosi semplicemente ai sassi della sponda e in alcuni casi tanto vistosa da sollevare il livello del lago fino a $30 \mathrm{~cm}$ e con periodo che va da 12 a 15 minuti. Anche a Bolsena si registrano sesse, ma, esse sono meno ampie che a Marta. Si riportano nella fig. 5, riprodotte a grandezza naturale, le belle registrazioni di sesse ottenute contemporaneamente a Marta e a Bolsena il 20 luglio 1903 dalle $13^{\text {h }}$ alle $21^{\text {th}}$. Dall'esame della registrazione ottenuta a Marta si vede che le ampiezze delle oscillazioni oltrepassano i $18 \mathrm{~cm}$ mentre a Bolsena si mantengono al di sotto di $9 \mathrm{~cm}$. Inoltre le registrazioni delle sesse a Marta sono continue, di una nitidezza particolare, generalmente molto regolari e possono raggiungere ampiezze tali da uscire fuori dal foglio del registratore. A Marta la sessa uninodale domina e finisce col mascherare le altre come è chiaramente visibile nella fig. 5 .

Dalla contemporanea registrazione ottenuta a Bolsena (fig. 5) si rileva come ivi le sesse siano meno vistose; però, oltre all'uninodale, si notano la binodale, la trinodale e, molto probabilmente, la quadrinodale.

Il particolare divario, relativo all'ampiezza delle sesse, nelle due 
località, viene confermato dalla teoria, come si vede dall'andamento delle sesse calcolate. Difatti col metodo di Hidaka (fig. 3) risulta che a Bolsena (sez. 24) l'ampiezza della sessa uninodale è circa il $70 \%$ di quella di Marta (sez. 0) e col metodo di Defant (fig. 4) lo scarto è ancora maggiore, in quanto la prima è il $55 \%$ della seconda.

La diversa natura della riva, ripida dalla parte di Bolsena e pianeggiante con poca profondità per un gran tratto a Marta, potrebbe giustificare tale differenza nell'ampiezza delle sesse.

Dallo specchietto seguente, dove sono posti a confronto i periodi medi delle tre sesse, rilevati dalle registrazioni del Palazzo (fig. 5), con quelli calcolati con i due metodi detti, si nota la perfetta concordanza tra i valori osservati e quelli teorici:

\begin{tabular}{|c|c|c|c|}
\hline \multicolumn{3}{|c|}{ P E R I O D I } & \\
\hline uninodale & binodale & trinodale & \\
& & & \\
\hline & & & \\
$13^{\mathrm{m}}, 05$ & $7^{\mathrm{m}}, 90$ & $5^{\mathrm{m}}, 40$ & \\
13,15 & 8,20 & 5,95 & metodo di Hidaka \\
13,23 & 7,92 & 5,40 & $\begin{array}{c}\text { ") " Defant } \\
\text { medi osservati } \\
\text { registrazioni Palazzo }\end{array}$
\end{tabular}

Nella registrazione di Bolsena si nota anche un periodo di $3^{\mathrm{m}}, 8$, che potrebbe corrispondere a quello della sessa quadrinodale. Dalla fig. 5 si rileva inoltre che vi è uno sfasamento di intensità tra il fenomeno registrato a Marta e a Bolsena, tanto che mentre nella registrazione di Marta la sessa è così ampia da provocare l'uscita della puntina scrivente dal foglio, in quella di Bolsena l'ampiezza della registrazione è scarsa per non dire nulla. Su questo fatto, ritorneremo in un lavoro successivo, dopo l'analisi di nuove registrazioni.

Dai risultati ottenuti in questo lavoro, si nota il pieno accordo tra i valori teorici e quelli dell'osservazione. Date le proporzioni veramente notevoli del fenomeno sareblye di sommo interesse poter eseguire una nuova serie di registrazioni contemporaneamente in più punti del lago, oltre a prove su un modello e, nel contempo, ampliare, la trattazione teorica. E quanto ci proponiamo di fare.

Roma - Istituto Nazionale di Geofisica - Dicembre 1950. 
Sesse contemporanee a MARTA ed a BOLSENA dalle ore 13 alle 21 del 20 Luglio 1903

Scala 1:1

\section{(1)}

a तs

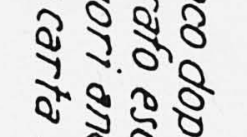

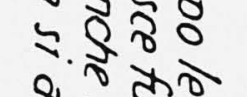

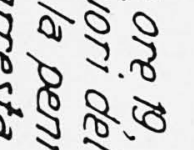

a

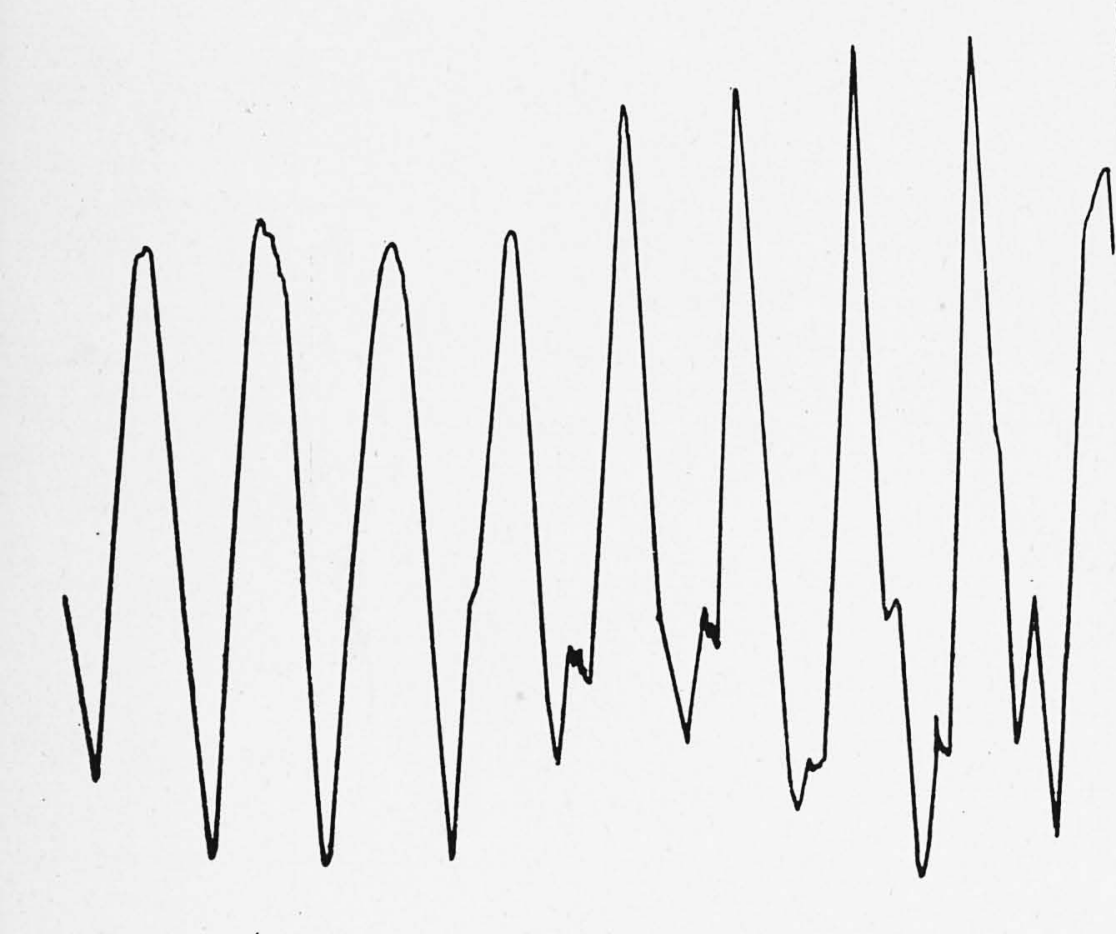
20
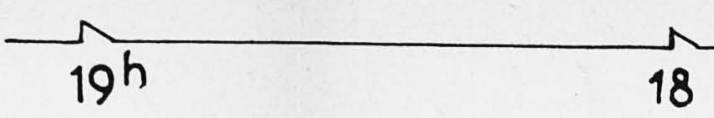

16

14
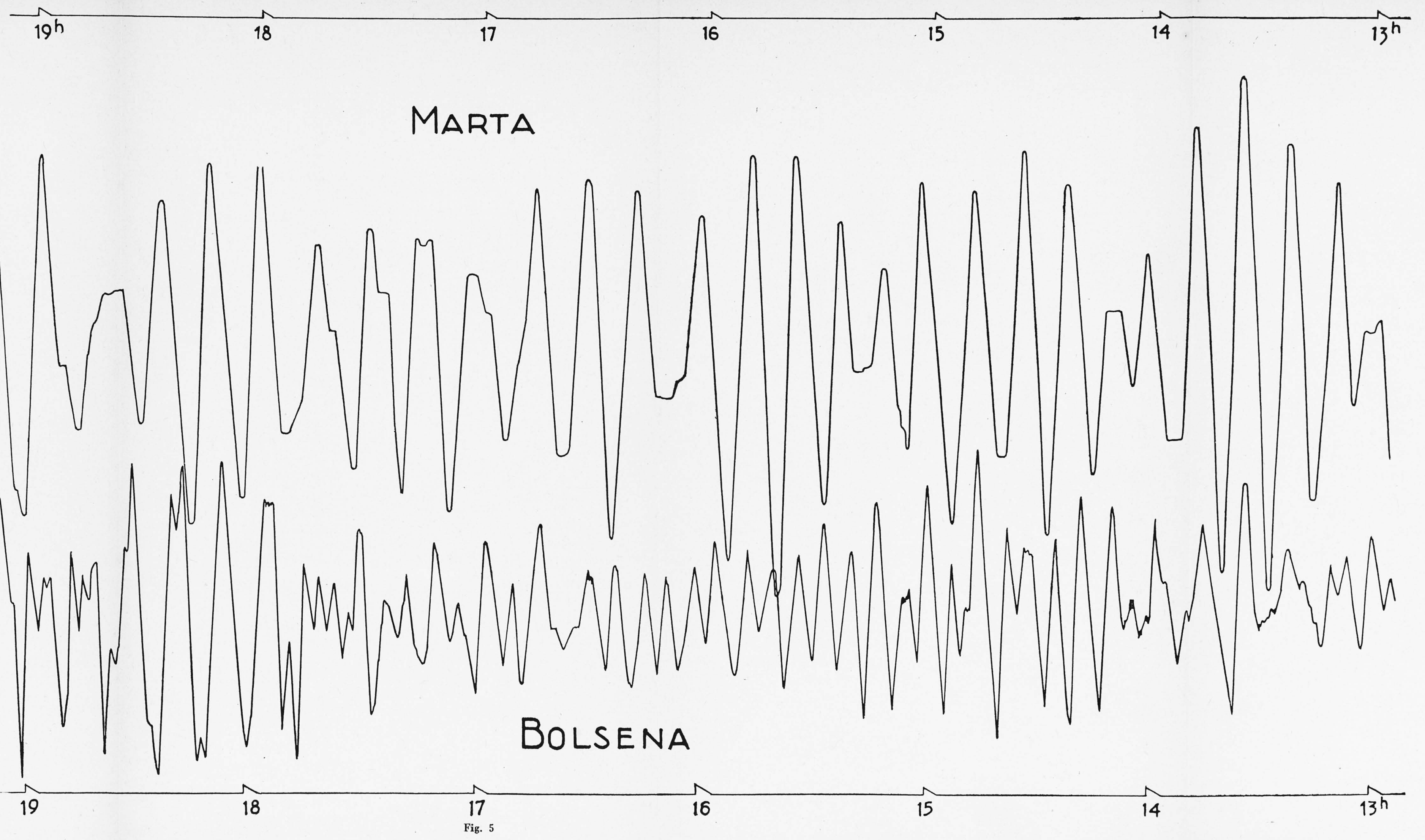


\section{RIASSUNTO}

Si pongono a confronlo $i$ risultati teorici degli elementi delle tre prime sesse del lago di Bolsena, calcolati con i metodi di Hidaka e di Defant, con quelli delle registrazioni ottenute contemporaneamente a Marta e a Bolsena da L. Palazzo il 20 luglio 1903. Viene constatata la perfetta concordanza tra gli uni e gli altri a convalida della bontà dei procedimenti teorici.

\section{BIBLIOGRAFIA}

(1) De Acostini G.: Esplorazioni idrografiche nei laghi vulcanici della provincia di Roma - Boll. Soc. Geograf. Ital. Vol. XI (1898).

(2) Patazzo L.: Studi limnologici sul lago di Bolscna - Atti del V Congresso Geografico Italiano. Vol. 2o. Sez. I (1904).

(3) Calor P.: Le sesse del lago di Garda. Parte I e II - Annali di Geofisica, n. 1 e 2 (1948). 